\title{
A new genus of metalmark moths (Lepidoptera, Choreutidae) with Afrotropical and Australasian distribution
}

\author{
Jadranka Rota ${ }^{1, \dagger}$, Scott E. Miller ${ }^{2, \ddagger}$ \\ I Laboratory of Genetics and Zoological Museum, Department of Biology, University of Turku, FI-20014 \\ Turku, Finland 2 National Museum of Natural History, Smithsonian Institution, P.O. Box 37012, Washing- \\ ton, D.C., 20013-7012, USA \\ † http://zoobank.org/F7481CDA-1995-468B-9AE5-AC1A9003805A \\ † http://zoobank.org/D70079F1-5135-46BA-A790-CB833B5892EF \\ Corresponding author: Jadranka Rota (jadranka.rota@utu.fi) \\ Academic editor: Alberto Zilli | Received 27 August 2013 | Accepted 7 October 2013 | Published 25 November 2013 \\ http://zoobank.org/B2DB1DE6-9291-4780-B483-2A31DC64B99F \\ Citation: Rota J, Miller SE (2013) A new genus of metalmark moths (Lepidoptera, Choreutidae) with Afrotropical and \\ Australasian distribution. ZooKeys 355: 29-47. doi: 10.3897/zookeys.355.6158
}

\begin{abstract}
Niveas Rota, new genus, and its two new species, N. agassizi Rota, new species, and N. kone Rota, new species, are described and illustrated. Niveas is assigned to the subfamily Choreutinae based on morphological and molecular data. Niveas agassizi is currently known only from Kenya and only from female specimens. Niveas kone has been found on the Solomon Islands and in Papua New Guinea (PNG). In PNG, larvae of this species have been reared from several species of Ficus (Moraceae). The two species are superficially quite dissimilar from each other. However, they share features in wing pattern and venation, as well as female genitalia, and the molecular data strongly support the monophyly of Niveas.
\end{abstract}

\section{Keywords}

Alpha taxonomy, DNA barcoding, Ficus spp., Kenya, Niveas agassizi, Niveas kone, Papua New Guinea, Solomon Islands, phylogenetics 


\section{Introduction}

Choreutidae, commonly known as metalmark moths, are a family of micro-moths with a worldwide distribution. The family is most species-rich in the tropics, and, as is the case for numerous other small tropical invertebrates, much of its richness is still unknown to science (unpublished data). Currently, 406 species of choreutids are described (Nieukerken et al. 2011).

Choreutids are medium-sized micro-moths with wingspans ranging from about one to two centimeters, often with bright colors and iridescent markings on their wings (Diakonoff 1986). They are diurnal with only some species attracted to lights at night (personal observation), making them a fairly rare group in museum collections. In our experience, large-scale rearing projects result in finding more species of choreutids than employing light traps.

Through exactly such efforts over the past 20 years in Papua New Guinea (PNG), the Binatang Research Center (BRC), with a large international group of collaborators focusing on the ecology of herbivorous insects and their host plants (Miller et al. 2003; Craft et al. 2010; Novotny et al. 2010; Hrcek et al. 2011; Hrcek et al. 2013; Miller et al. 2013), the number of known species of choreutids and our knowledge of their biology have greatly increased. One of the many new species of choreutids found in PNG during this project is sufficiently different from all described species that it requires a new genus.

Coincidentally, through separate collecting efforts by David Agassiz in Africa, a related species was discovered in Kenya. Herein these two species, as well as the genus to which they belong, are described and illustrated, and the phylogenetic position of the new genus within the family is discussed.

The shared presence of the terminal black band with white spots in the forewing (arrows in Figs 1, 3) was the first indication that N. kone Rota, sp. n. and N. agassizi Rota, sp. n. might be related. Initially this relationship seemed unlikely because of the disjunct geographical distribution of the two ( $N$. kone being distributed in the Australasian Region and $N$. agassizi in the Afrotropical Region) and because their DNA barcodes did not suggest a close relationship. However, once the similarities in wing venation and female genitalia were noticed, and we included nuclear genes in the analysis with a more extensive choreutid molecular dataset, the results strongly supported the close relationship between $N$. kone and $N$. agassizi.

\section{Methods}

All material examined is listed in Table 1. Layered photographs of specimens and slides were taken using an Olympus SZX16 microscope with motorized focus drive attached to an Olympus E520 digital camera. The photographs were then combined by using the programs Deep Focus 3.1 and Quick Photo Camera 2.3. The wing venation drawing was made digitally in Adobe Illustrator CS3 overlaid on top of a slide photograph. 


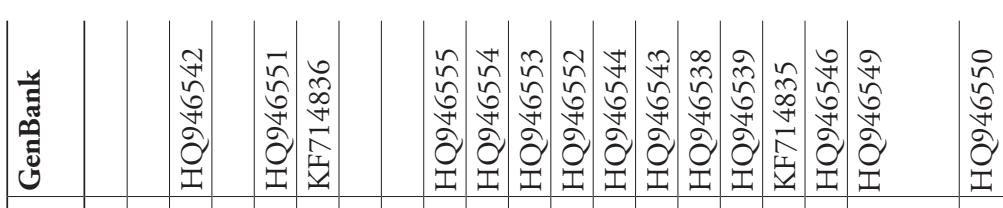
क

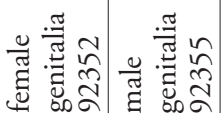

\begin{tabular}{|c|c|c|c|c|c|c|c|c|c|c|c|c|c|c|c|c|c|c|c|c|c|c|c|c|}
\hline 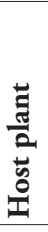 & 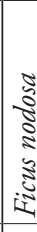 & 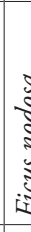 & 过 & & | & 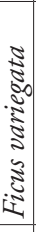 & క్ & 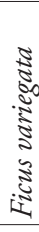 & 3 & 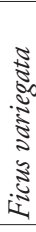 & 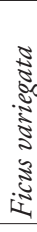 & 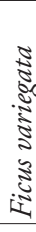 & 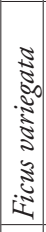 & 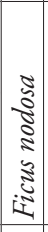 & 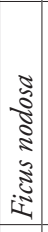 & 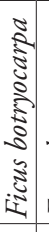 & 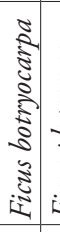 & 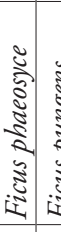 & 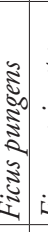 & 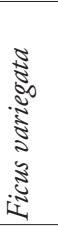 & 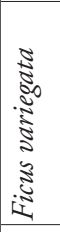 & 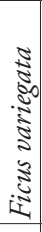 & 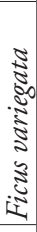 & 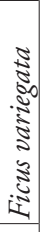 \\
\hline 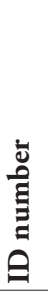 & 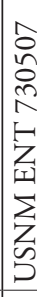 & 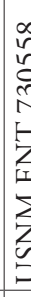 & $\left\{\begin{array}{lll}1 \\
1 \\
1 \\
1 \\
1\end{array}\right.$ & & 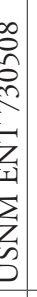 & 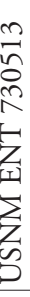 & 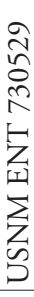 & 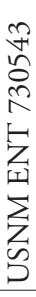 & $\sum_{i}$ & 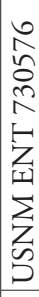 & 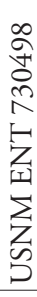 & 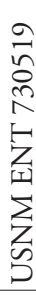 & 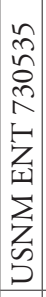 & 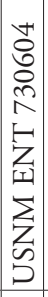 & 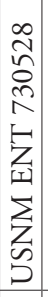 & 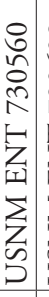 & 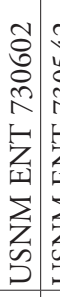 & 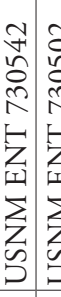 & 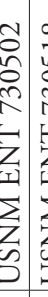 & 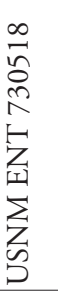 & 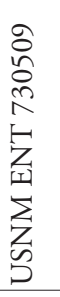 & 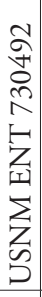 & 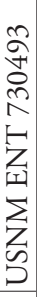 & 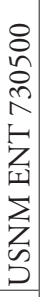 \\
\hline $\begin{array}{l}\stackrel{0}{0} \\
\overline{0} \\
\dot{0}\end{array}$ & $\frac{\pi}{\infty}$ & 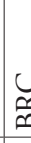 & & 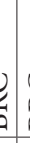 & 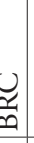 & 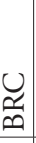 & $\frac{2}{\infty}$ & $\stackrel{\mathscr{m}}{\mathscr{n}}$ & $\frac{\mathscr{v}}{m}$ & $\frac{U}{\mathscr{n}}$ & 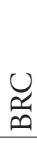 & $\begin{array}{l}u \\
\frac{1}{\infty}\end{array}$ & 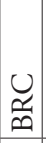 & 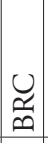 & $\mid \begin{array}{l}u \\
\frac{x}{\infty} \\
\end{array}$ & 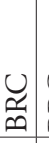 & $\underset{\sim}{\mathscr{n}}$ & $\stackrel{\sim}{\infty}$ & 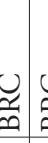 & $\frac{\mathscr{x}}{\mathscr{\infty}}$ & $\begin{array}{l}\mathscr{x} \\
\stackrel{x}{\infty}\end{array}$ & $\begin{array}{l}0 \\
\stackrel{x}{\infty}\end{array}$ & $\begin{array}{l}0 \\
\frac{1}{\infty} \\
\end{array}$ & $\frac{\mathscr{\sim}}{\mathscr{n}}$ \\
\hline ڤี๊ & $\stackrel{\curvearrowleft}{\precsim}$ & $\frac{v}{2}$ & $\frac{1}{\alpha}$ & & $\begin{array}{l}\sigma \\
\sigma \\
\\
\delta \\
\delta\end{array}$ & 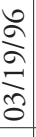 & $\frac{n}{\hat{\sigma}}$ & 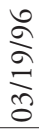 & $\stackrel{\curvearrowright}{2}$ & 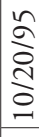 & 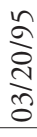 & 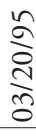 & 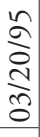 & $\begin{array}{l}\tilde{n} \\
\hat{a} \\
\frac{n}{2} \\
\hat{\sigma}\end{array}$ & 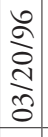 & 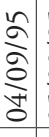 & 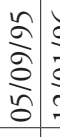 & 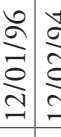 & 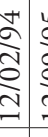 & 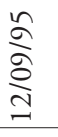 & $\frac{n}{\hat{\sigma}}$ & 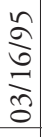 & 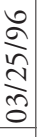 & $\frac{\tilde{\sigma}}{\hat{\sigma}}$ \\
\hline لَّ & 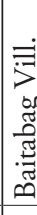 & & & & صี & 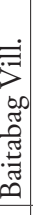 & & 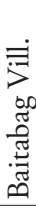 & & 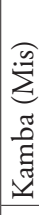 & 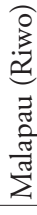 & 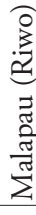 & 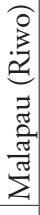 & 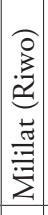 & 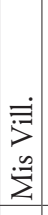 & 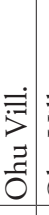 & 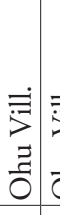 & 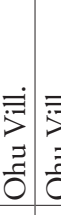 & 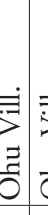 & $\begin{array}{l}\bar{J} \\
\overrightarrow{3} \\
\overline{0}\end{array}$ & $\begin{array}{l}\bar{\Xi} \\
\overrightarrow{3} \\
\vec{\Xi} \\
0\end{array}$ & 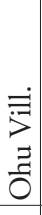 & 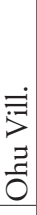 & 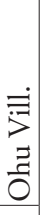 \\
\hline 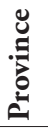 & $\pi_{i}^{\pi}$ & & & & $\dot{\Sigma}$ & 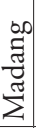 & & 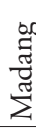 & & $\frac{\pi}{\pi}$ & $\begin{array}{l}\frac{\mathrm{J}}{0} \\
\sum^{\pi}\end{array}$ & 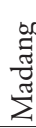 & $\begin{array}{l}\infty \\
\frac{0}{\pi} \\
\frac{\pi}{2}\end{array}$ & $\begin{array}{l}\infty \\
\frac{5}{5} \\
\frac{\pi}{\pi} \\
\Sigma \\
\Sigma\end{array}$ & 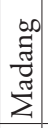 & 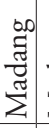 & 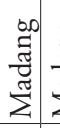 & 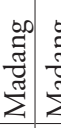 & 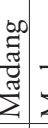 & 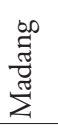 & 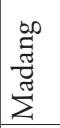 & 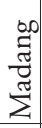 & 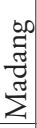 & 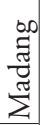 \\
\hline
\end{tabular}

要

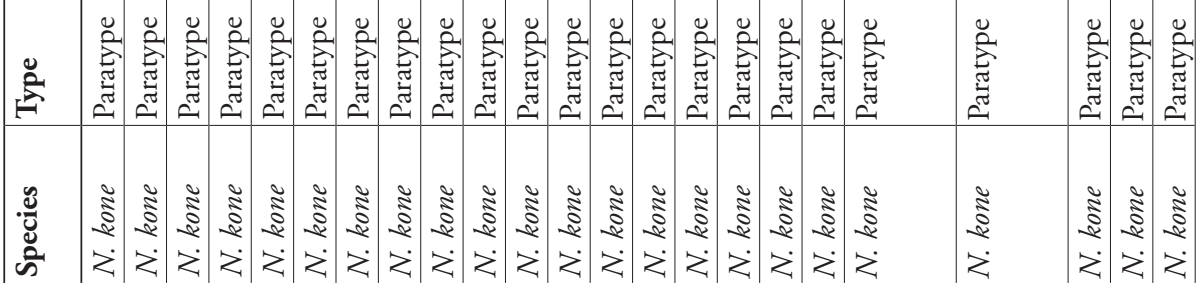




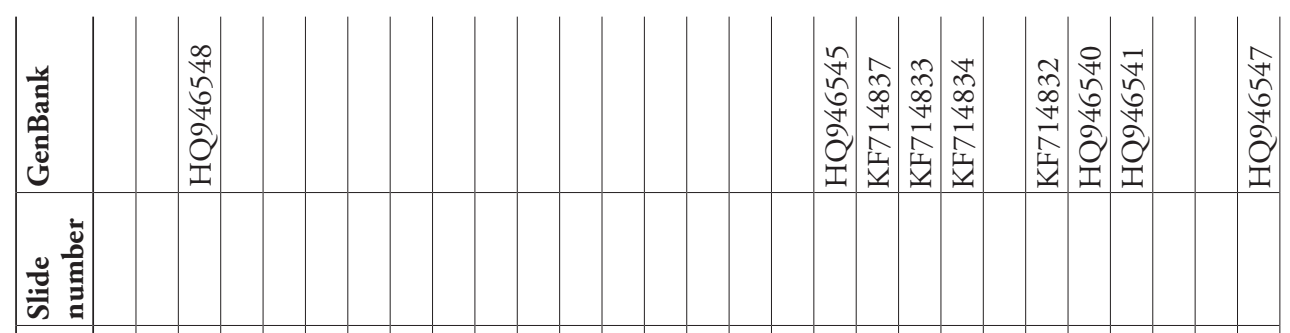

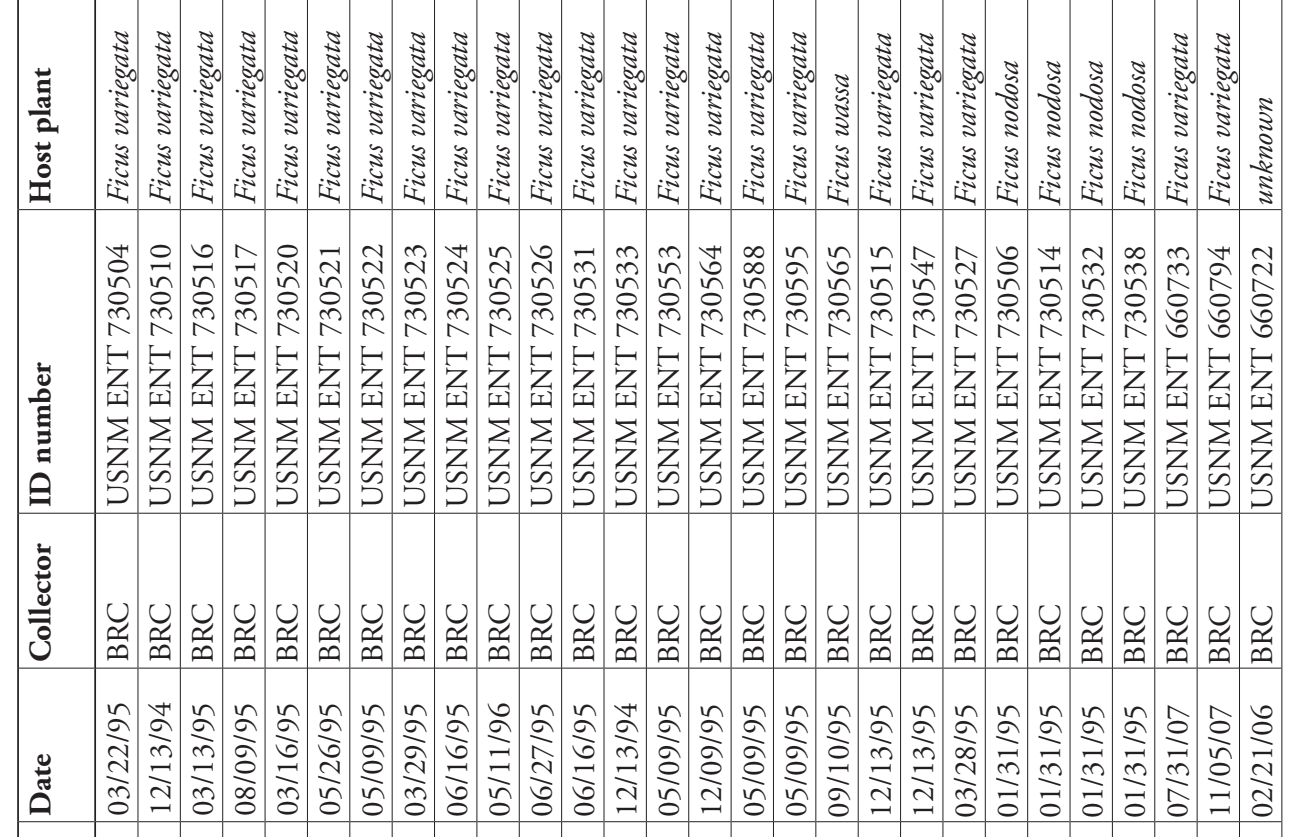

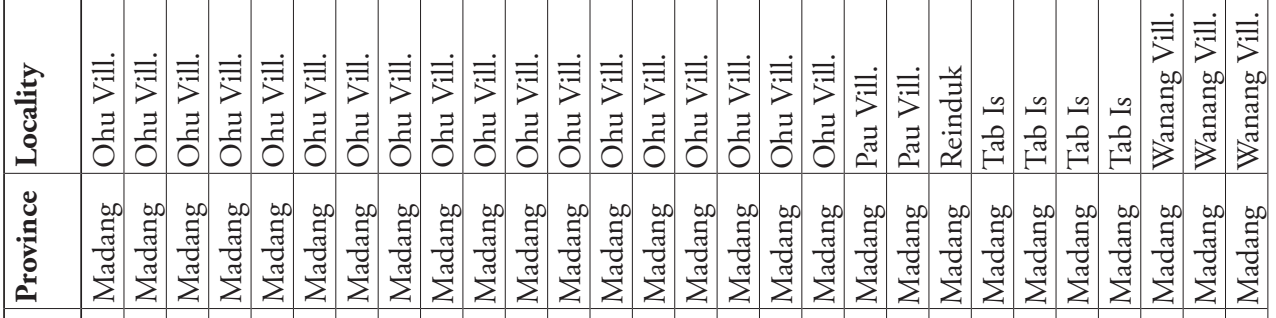

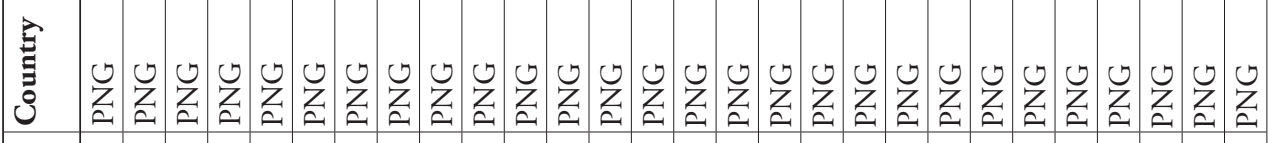

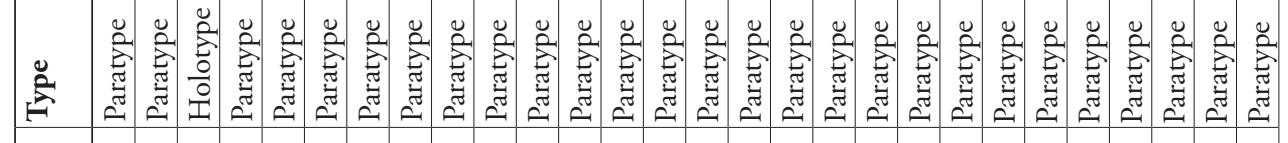

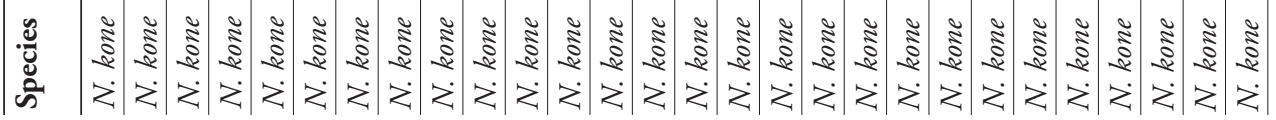




\begin{tabular}{|c|c|c|c|c|c|c|c|c|c|}
\hline 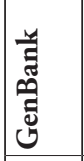 & & & & 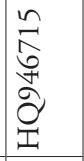 & 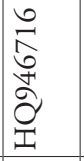 & & & & \\
\hline 营 & 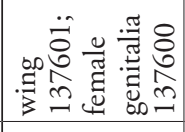 & & & & & 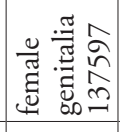 & 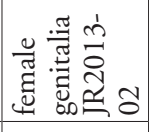 & 皇 & 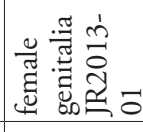 \\
\hline 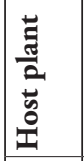 & 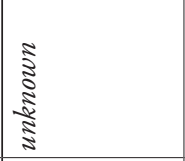 & 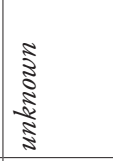 & 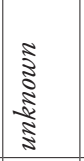 & 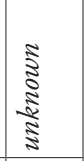 & 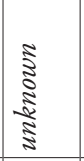 & 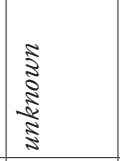 & $\begin{array}{c}5 \\
\frac{3}{2} \\
5 \\
5 \\
5 \\
5\end{array}$ & 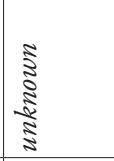 & 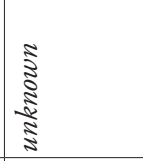 \\
\hline 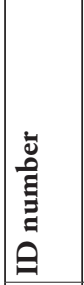 & 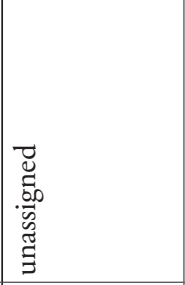 & 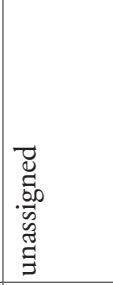 & 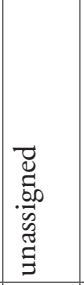 & 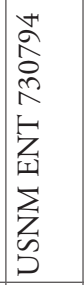 & 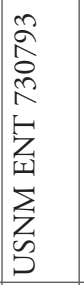 & 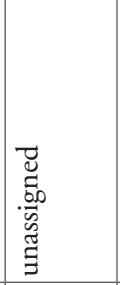 & 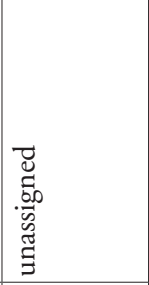 & 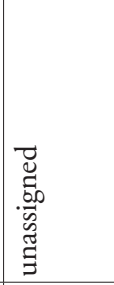 & 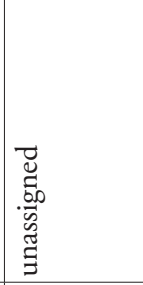 \\
\hline 苞 & 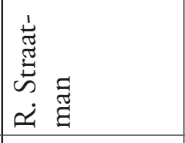 & 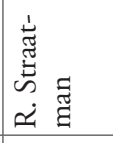 & 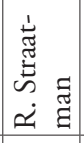 & 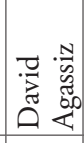 & 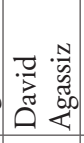 & 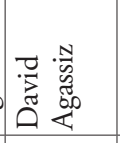 & 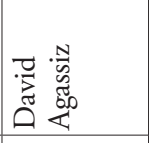 & 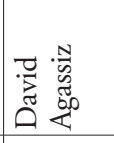 & 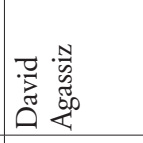 \\
\hline ڤั๊ & $\frac{\sqrt{5}}{n}$ & $\frac{\sqrt[c]{0}}{\frac{m}{n}}$ & $\begin{array}{l}\frac{1}{0} \\
\frac{n}{2} \\
\infty \\
0\end{array}$ & $\frac{8}{\stackrel{8}{a}}$ & $\frac{8}{\stackrel{8}{2}}$ & 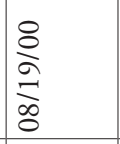 & 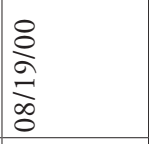 & 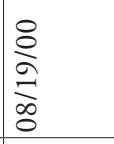 & $\begin{array}{l}8 \\
\stackrel{8}{9} \\
\stackrel{1}{\infty} \\
\infty \\
0\end{array}$ \\
\hline 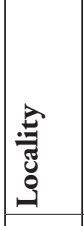 & 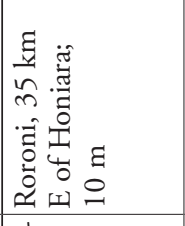 & 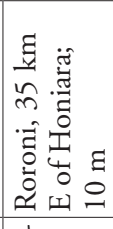 & 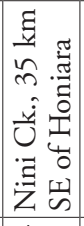 & 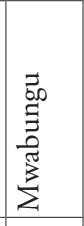 & 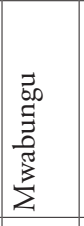 & 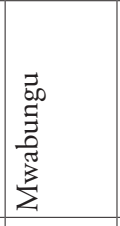 & 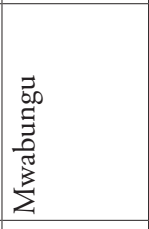 & 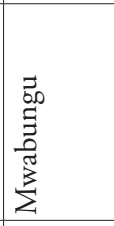 & 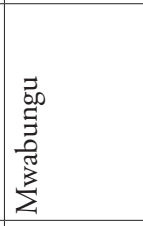 \\
\hline 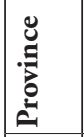 & 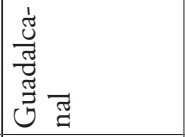 & 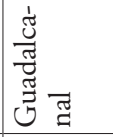 & 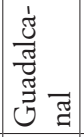 & $\begin{array}{ll}0 & \frac{0}{\pi} \\
0 & \frac{3}{3} \\
0 & \frac{3}{4} \\
0\end{array}$ & 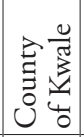 & 㲵造 & 竞造 & 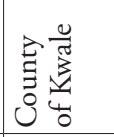 & 总告 \\
\hline 递 & $\begin{array}{l}\dot{m} \\
\ddot{0} \\
\dot{\Xi} \\
0 \\
\dot{0} \\
\dot{n}\end{array}$ & $\begin{array}{l}\dot{\oplus} \\
\overrightarrow{0} \\
\stackrel{0}{g} \\
0 \\
\dot{0} \\
\dot{0}\end{array}$ & 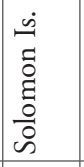 & 㺃 & 㺃 & 泀 & 泀 & 苞 & 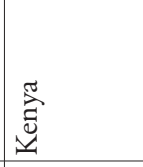 \\
\hline 念 & 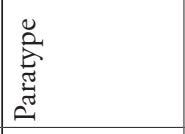 & 莺 & 总 & 空 & 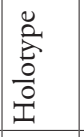 & 总 & 总 & $\sum_{\substack{\tilde{n}\\
\\
}}^{0}$ & 总 \\
\hline 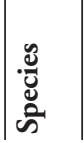 & \begin{tabular}{|l}
$z$ \\
$\vdots$ \\
0 \\
$z$ \\
$z$
\end{tabular} & 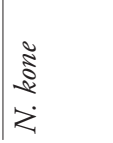 & $\mid$ & 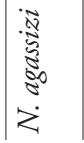 & $\begin{array}{l}-\bar{N} \\
2 \\
5 \\
8 \\
\vdots \\
z\end{array}$ & 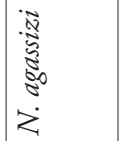 & $\begin{array}{l}\bar{N} \\
3 \\
5 \\
8 \\
\vdots \\
z\end{array}$ & 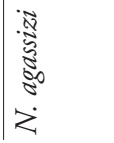 & $\begin{array}{l}-\overline{2} \\
\tilde{2} \\
8 \\
8 \\
z \\
z\end{array}$ \\
\hline
\end{tabular}


All images were improved in Adobe Photoshop CS3. Genitalic dissections and terminology follow Rota (2008b).

Field sampling and rearing protocols for the PNG material are detailed in Miller et al. (2003; 2013), Craft et al. (2010), and Novotny et al. (2010). The Plant List website (2010) was used for host plant names. Latitude, longitude, and altitude data for the collecting localities is in Table 2.

The molecular phylogeny dataset included three outgroups and 40 species of ingroup taxa, including two individuals each of Niveas kone and $N$. agassizi totaling 45 terminal units. We analyzed data from eight genes: COI (mitochondrial), CAD, EF1 $a$, GAPDH, IDH, MDH, RpS5, and wingless (all nuclear) (Wahlberg and Wheat 2008). The final alignment was 6187 base pairs long. Molecular sequences for all taxa except N. kone and N. agassizi are from Rota (2011) and Rota and Wahlberg (2012), and their GenBank accession numbers can be found there. For the specimens of $N$. kone (660733) and $N$. agassizi (Ch_JR44_1), DNA extraction was done from whole abdomens, which were later used for dissection of genitalia. Because the DNA amplification methods described by Wahlberg and Wheat (2008) did not work for obtaining sequences of nuclear genes from these specimens, suggesting that their DNA was too degraded for the standard approach, we used newly-designed primers (Niklas Wahlberg, unpublished) (Table 3) to amplify short fragments of the nuclear genes (see Table 4 for total number of base pairs for each gene fragment amplified and the GenBank accession numbers for fragments longer than 200 base pairs). For sequence storage and manipulation we used the VoSeq application (Peña and Malm 2012). The nexus file with the alignment is available from the Figshare Digital Repository: doi: 10.6084/m9.figshare.811841

Both maximum likelihood (ML) and Bayesian phylogenetic analyses were performed. ML analysis of unpartitioned data was conducted using RAxML blackbox available online (Stamatakis et al. 2008) with the GTR+G model and 100 bootstraps. Bayesian analysis of data partitioned using the program TIGER (Cummins and McInerney 2011) as described in Rota and Wahlberg (2012) was carried out in MrBayes v. 3.2 (Ronquist et al. 2012) for 10 million generations with one cold and three heated chains, sampling trees every 1000 generations. The analyses were run on the freely available Bioportal server (University of Oslo, Norway). The convergence was assessed

Table 2. Locality information.

\begin{tabular}{l|c|c|c}
\hline Locality & m.a.s.l. & latitude & longitude \\
\hline Baitabag village \& Kau Wildlife Area, near Madang, Madang Province, PNG & 50 & $\mathrm{~S}^{\circ} 08^{\prime}$ & $\mathrm{E} 145^{\circ} 46^{\prime}$ \\
\hline Mis, Madang Province, PNG & 50 & $\mathrm{~S}^{\circ} 11^{\prime}$ & $\mathrm{E} 145^{\circ} 47^{\prime}$ \\
\hline Ohu Conservation Area, Ohu village near Gum river, Madang Province, PNG & 100 & $\mathrm{~S} 5^{\circ} 13^{\prime}$ & $\mathrm{E} 145^{\circ} 41^{\prime}$ \\
\hline Pau, Madang Province, PNG & 0 & $\mathrm{~S} 5^{\circ} 08^{\prime}$ & $\mathrm{E} 145^{\circ} 46^{\prime}$ \\
\hline Reinduk, Madang Province, PNG & 225 & $\mathrm{~S} 5^{\circ} 39^{\prime}$ & $\mathrm{E} 145^{\circ} 24^{\prime}$ \\
\hline Riwo, Madang Province, PNG & 0 & $\mathrm{~S}^{\circ} 09^{\prime}$ & $\mathrm{E} 145^{\circ} 48^{\prime}$ \\
\hline Tab Island, Madang Province, PNG & 0 & $\mathrm{~S} 5^{\circ} 10.6^{\prime}$ & $\mathrm{E} 145^{\circ} 52.6^{\prime}$ \\
\hline Wanang village, Madang Province, PNG & 115 & $\mathrm{~S} 5^{\circ} 13.9^{\prime}$ & $\mathrm{E} 145^{\circ} 10.9^{\prime}$ \\
\hline Mwabungu, County of Kwale, Kenya & 0 & $\mathrm{~S} 4^{\circ} 20.3^{\prime}$ & $\mathrm{E} 39^{\circ} 37^{\prime}$ \\
\hline
\end{tabular}


by examining plots of log likelihoods and all model parameters using Tracer v.1.5 (Rambaut and Drummond 2007), as well as potential scale reduction factors and split frequencies, both reported by MrBayes. Branch support is expressed as Bayesian posterior probability (PP) and maximum likelihood bootstraps (ML BS).

DNA barcode sequences (COI) for Niveas kone (24 specimens) and Niveas agassizi (2 specimens) were obtained at the Biodiversity Institute of Ontario, University of

Table 3. Primers.

\begin{tabular}{l|l}
\hline COI-1F & GGTCAACAAATCATAAAGATATTGG \\
\hline COI-1R & GGWGCYCCTARTATTAAAGGWAYTA \\
\hline EF-1F & CACATYAACATTGTCGTSATYGG \\
\hline EF-1R & TRSCGGTYTCGAACTTCCA \\
\hline EF-2F & GAGCGTGARCGTGGTAT \\
\hline EF-2R & RGCTTCGAACTCACCRGTA \\
\hline EF-3F & TCAAGAACATGATCACYGG \\
\hline EF-7F & GARGAYACTTCCTTCTTGA \\
\hline EF-8R & CAAYGTTGGTTTCAACGT \\
\hline GAPDH-1F & ACAGCVACKGTYTGYCTCATRTC \\
\hline GAPDH-1R & AARGCTGGRGCTGAATATGT \\
\hline GAPDH-2F & AAGTTGTCATGGATRACCTT \\
\hline GAPDH-2R & TAACTTTGCCRACAGCYTT \\
\hline GAPDH-3F & GTGCCCARCARAACATCAT \\
\hline GAPDH-3R & TCAGCGGCTTCCTTRACCT \\
\hline IDH-1F & GGWGAYGARATGACNAGRATHATHTGG \\
\hline IDH-1R & GGACTCTTCCACATTTTYTT \\
\hline MDH-1F & GAYATNGCNCCNATGATGGGNGT \\
\hline MDH-1R & TCYTTRCGRGCAACYTTRTC \\
\hline RPS5-1F & ATGGCNGARGARAAYTGGAAYGA \\
\hline RPS5-1R & TTGTGWGCRTACCTRCCRGC \\
\hline
\end{tabular}

Table 4. GenBank accession numbers and the number of base pairs for each gene fragment.

\begin{tabular}{l|c|c|c|c}
\hline & N. agassizi (730793) & N. agassizi (Ch_JR44_1) & N. kone (730509) & N. kone (660733) \\
\hline \multirow{2}{*}{ COI } & HQ946716 & - & HQ946550 & KF646130 \\
\cline { 2 - 5 } & $609 \mathrm{bp}$ & $176 \mathrm{bp}$ & $658 \mathrm{bp}$ & $610 \mathrm{bp}$ \\
\hline \multirow{2}{*}{ EF1 $a$} & - & KF646128, KF646129 & - & $\begin{array}{c}\text { KF646131, } \\
\text { KF646132 }\end{array}$ \\
\cline { 2 - 5 } & - & $550 \mathrm{bp}$ & - & $706 \mathrm{bp}$ \\
\hline GAPDH & - & - & - & KF646133 \\
\hline IDH & - & $136 \mathrm{bp}$ & - & $430 \mathrm{bp}$ \\
\hline MDH & - & $135 \mathrm{bp}$ & - & - \\
\hline RpS5 & - & $190 \mathrm{bp}$ & - & - \\
\hline
\end{tabular}


Guelph, using their standard methodology (Craft et al. 2010; Hrcek et al. 2011; Wilson 2012). They are deposited in GenBank as accessions listed in Table 1, and their full data including images are in the Barcode of Life Database (http://www.boldsystems. org; see Ratnasingham and Hebert 2007; 2013). These sequences were also analyzed with MrBayes v. 3.2 (unpartitioned dataset, 2 million generations).

\section{Results}

\section{Taxonomy}

Niveas Rota, gen. $\mathbf{n}$.

http://zoobank.org/F352952E-0F21-464F-BD1E-278C9A0679C1

http://species-id.net/wiki/Niveas

Figs 1-9

Type species. Niveas kone.

Material examined. See Table 1.

Distribution. Kenya, Papua New Guinea, Solomon Islands.

Diagnosis. Niveas can be easily distinguished from most genera of choreutids by the wing pattern (Figs 1,3). Superficially, species of Niveas are similar to some species of Anthophila and Choreutis, but there is no known species in either of the latter two genera with a black terminal band enclosing white spots in the forewing as in $N$. agassizi and $N$. kone. (Figs 1,3 ). Forewing venation with only four radial branches or with $\mathrm{R}_{4}$ and $\mathrm{R}_{5}$ fused in the basal half is also diagnostic for the genus. Female genitalia with paired concave sclerotizations on A7 sternite are also unique to Niveas.

Description. Head. Labial palpi with projecting ventral scale tufts (Figs 2, 4). Wings. Forewing veins $\mathrm{R}$ four-branched in $N$. kone (Fig. 5), five-branched in $N$. agassizi (Fig. 6), with $\mathrm{R}_{4}$ an $\mathrm{R}_{5}$ fused in basal 3/5; CuP present at termen for $1 / 3$ to $1 / 5$ wing length, extending as fold further towards base. Hindwing ten-veined, with $\mathrm{M}_{2}$ in close proximity to the basally fused $\mathrm{M}_{3}$ and $\mathrm{CuA}_{1}(N$. agassizi) or nine-veined, apparently with $\mathrm{M}_{3}$ and $\mathrm{CuA}_{1}$ completely fused into a single vein (Figs 5, 6). Male genitalia. Tegumen rounded on top, tuba analis extending beyond tegumen; vinculum as inverted trapezoid ventrally emarginate; valva with costal margin straight, ventral margin rounded, ending with a horn-like projection; phallus twice as long as valva (Fig. 7). Female genitalia. Apophyses anteriores slightly longer than posteriores; ostium bursae on A7 with a more or less strongly sclerotized antrum; ductus bursae straight, not coiled, with strong lateral sclerotizations; corpus bursae as a single sac ( $N$. agassizi) or divided into two sacs $(N$. kone) with one or more signa. A7 sternite with paired, somewhat rounded, concave sclerotizations proximally, clearly visible in $N$. kone (Fig. 8), and slightly less so in $N$. agassizi (Fig. 9).

Host plants. Genus Ficus (Moraceae). 


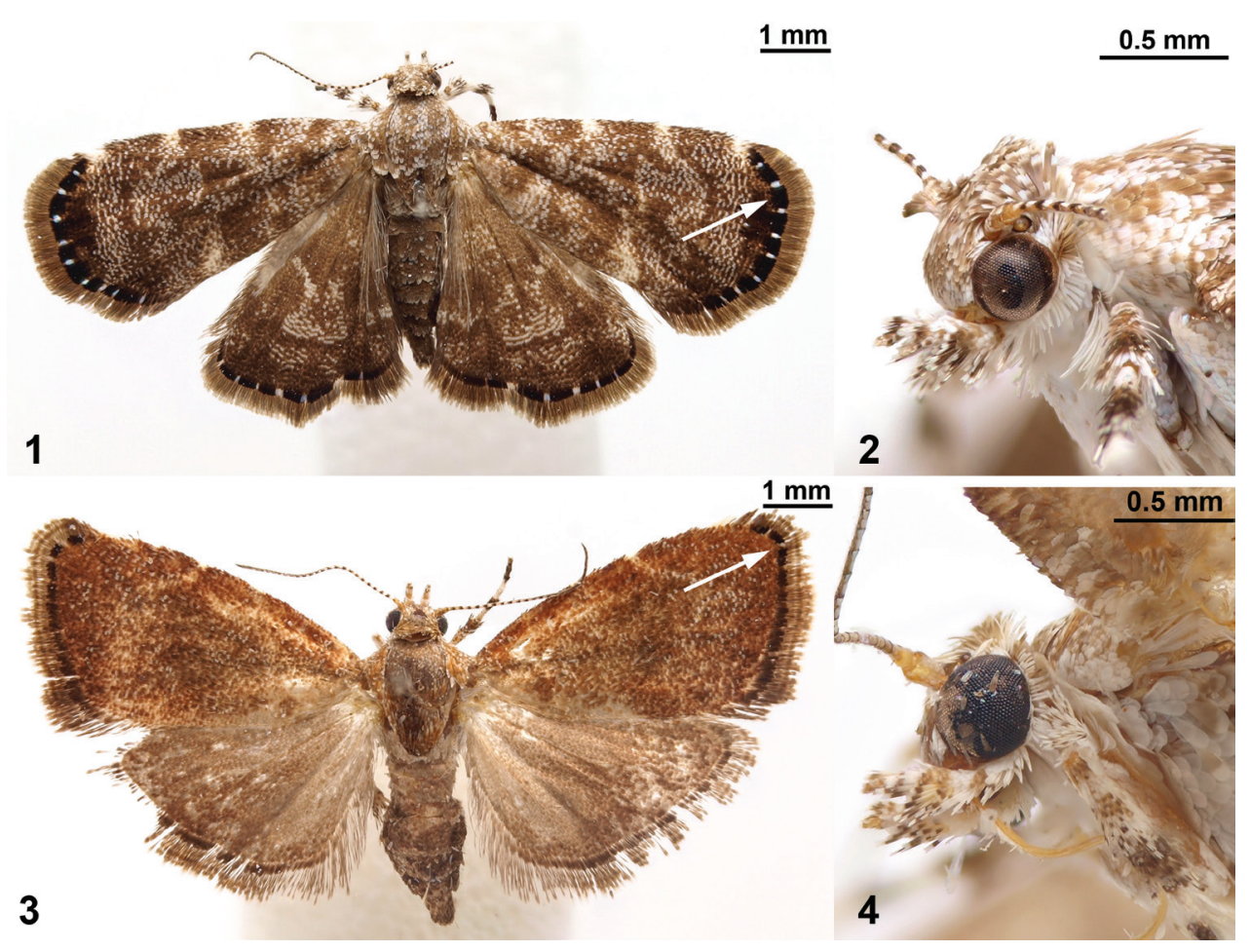

Figures I-4. Niveas kone: I Habitus 2 Head. Niveas agassizi: 3 Habitus 4 Head. (In Figs 1 and 3 arrows point at the terminal black band enclosing white spots.)

Etymology. The generic name is derived from Latin niveum, meaning snowy, in reference to speckles of white-tipped scales in the wings of the type species; it is not treated as a Latin word and is feminine in gender.

\section{Niveas kone Rota, sp. n.}

http://zoobank.org/9EA367B0-6B92-48FA-8075-D8D0D0BFA566 http://species-id.net/wiki/Niveas_kone

Figs $1,2,5,7,8$

Material examined. See Table 1.

Material deposited. The holotype and most paratypes will be retained at USNM, with paratypes distributed to PNG National Agriculture Research Institute (Port Moresby), BMNH, Bishop Museum, Naturalis (Leiden), and CSIRO (Canberra).

Distribution. Papua New Guinea, Solomon Islands.

Diagnosis. $N$. kone can be separated from all other known choreutids based on its wing pattern (Fig. 1). Superficially, it is similar to a few species of Brenthia Clemens, 1860 and Litobrenthia Diakonoff, 1978 owing to its background color, but it lacks iridescent 
spots along forewing termen, which are always present in those two genera. Both male and female genitalia are very distinct from those of other choreutids (Figs 7, 8).

Description. Head. Fig. 2. Wings. Fore- and hindwing with brown background color, speckled with white-tipped scales in an irregular pattern; a distinct black band along termen of both wings within which are more or less equidistant white spots (Fig. 1). Male genitalia. As for the genus (Fig. 7). Female genitalia. Corpus bursae split
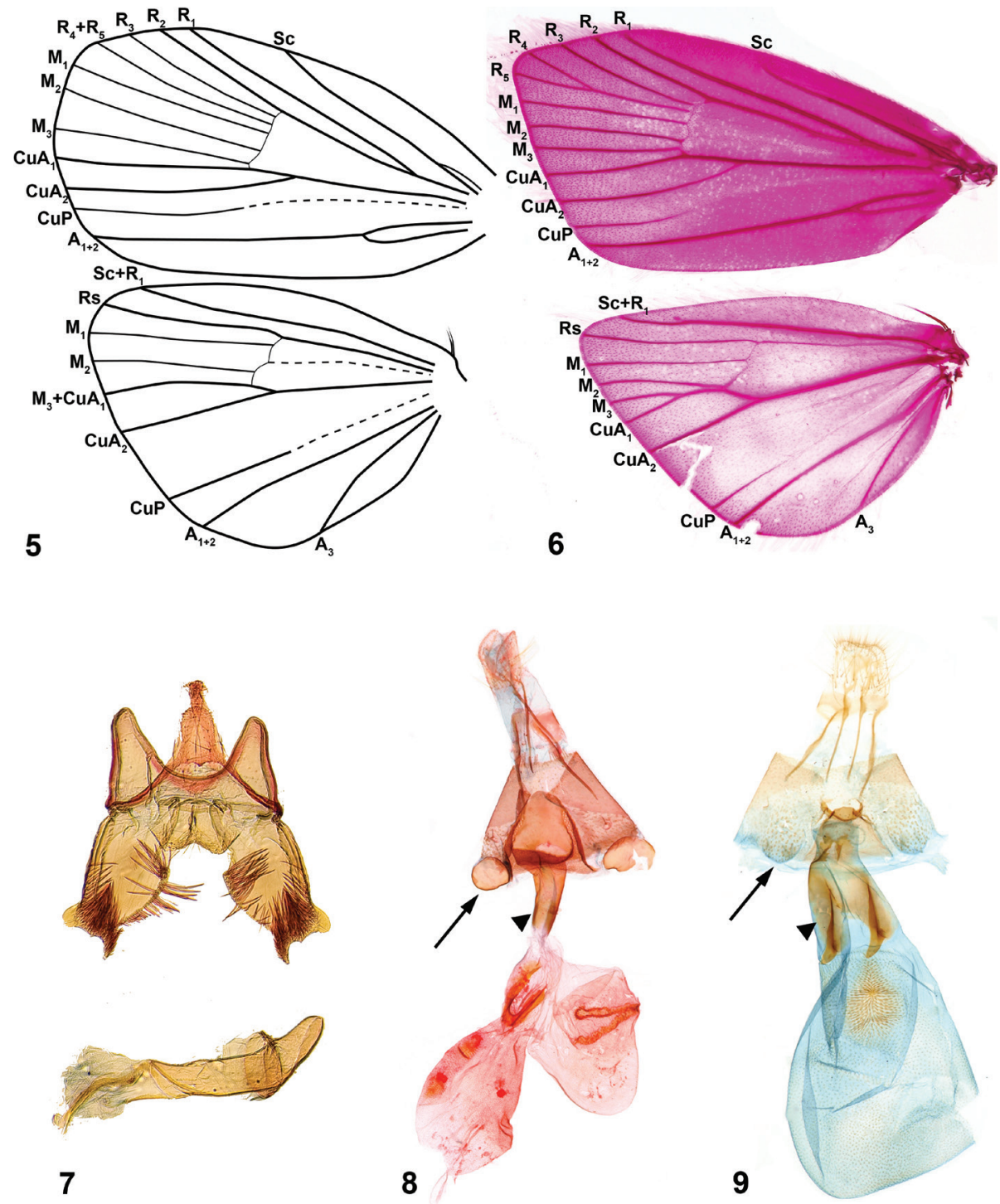

Figures 5-9. Niveas kone: 5 Wing venation 7 Male genitalia 8 Female genitalia. Niveas agassizi: 6 Wing venation 9 Female genitalia. (In Figs 8 and 9 arrows point at the A7 sternite sclerotizations, and triangles point at the lateral sclerotizations on the ductus bursae.) 
into two sacs; one sac with a V-shaped signum, the other with two round signa (Fig. 8). Immature stages. Fig. 12. See a brief note in text.

Host plants. Ficus botryocarpa Miq., F. nodosa Teijsm. \& Binn., F. phaeosyce K. Schum. \& Lauterb., F. pungens Reinw. ex Blume, F. variegata Blume, and F. wassa Roxb. (Moraceae).

Etymology. The species is named after the Finnish Kone Foundation (Koneen Säätiö) in appreciation of their funding of this work. The name is a noun in apposition.

\section{Niveas agassizi Rota, sp. n.}

http://zoobank.org/7F08322B-C0D2-450C-9DFF-ED9E4FEA5892

http://species-id.net/wiki/Niveas_agassizi

Figs 3, 4, 6, 9

Material examined. See Table 1.

Material deposited. The holotype will be deposited in National Museums of Kenya (Nairobi) (NMK), with paratypes to USNM, BMNH and NMK.

Distribution. Kenya.

Diagnosis. $N$. agassizi can be separated from other known choreutids by the wing pattern (Fig. 3). It is superficially similar to some species of Choreutis, but the latter usually have forewings with apparent patterning, and this is absent in $N$. agassizi. Female genitalia are very distinct from those of other choreutids (Fig. 9).

Description. Male unknown. Head. Fig. 4. Wings. Forewing bronze-brown with speckled white-tipped scales over most of its surface; distinct dark brown to black band along termen with two small white spots at apex; hindwing light brown (Fig. 3). Male genitalia. Unknown. Female genitalia. Ductus bursae short and wide, opening into large corpus bursae, with one oval signum (Fig. 9). Immature stages. Unknown.

Host plants. Unknown.

Etymology. This species is named after David Agassiz, who collected all the known specimens and made many significant contributions to our knowledge of African micro-moths. The name is a noun in the genitive case.

\section{Remarks}

We obtained 19 full-length barcodes of Niveas kone, as well as 5 shorter fragments. These form cluster AAB7478 in the Barcode of Life Database (accessed 29 August 2013), and using the RESL algorithm as implemented there (Ratnasingham and Hebert 2013), the maximum distance between the COI sequences for members of the species is $0.65 \%$, whereas the distance to the nearest cluster ( $N$. agassizi) is $9.22 \%$. In a Bayesian analysis of the COI sequences, all $N$. kone and all $N$. agassizi specimens grouped together with the other members of their species with very high branch support $(\mathrm{PP}=1)($ Fig. 10). 


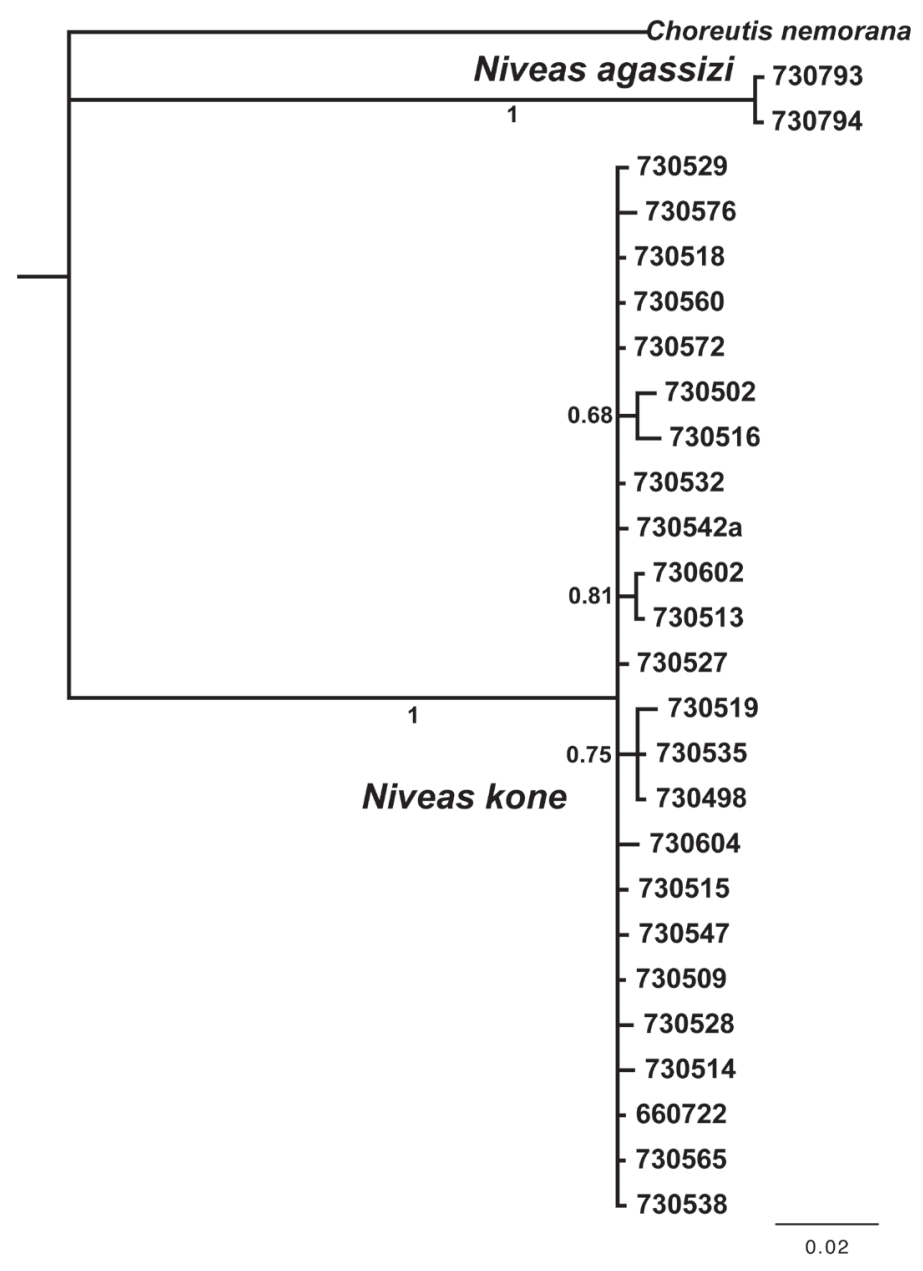

Figure 10. DNA barcode tree from a Bayesian analysis showing low divergence within species and high between species of Niveas. Numbers below or next to branches are Bayesian posterior probabilities. Specimen ID numbers are used as labels for the terminal branches.

The placement of Niveas in the choreutid generic phylogeny is very strongly supported. Niveas clearly belongs within the subfamily Choreutinae ( $\mathrm{PP}=1 ; \mathrm{ML} B S=96)$, and it appears to be the sister group of Choreutis ( $\mathrm{PP}=1.00$; ML BS=92) (Fig. 11).

Further comments on the biology of Niveas kone: Over the years, BRC field teams have encountered larvae identified as Niveas kone (as project morphospecies TORT015) 118 times, of which 62 were reared to adults, usually on Ficus nodosa and Ficus variegata, but also on four other species of Ficus (see full host plant list under $N$. kone description). Larvae have been found in all months except April and November, and are described by BRC staff as being green-clear-whitish in color, with short white hairs, and one spot on the side of the head (Fig. 12). Larvae of $N$. kone share the presence of short hairs with other Choreutinae (Rota 2005), which is unlike Brenthiinae 


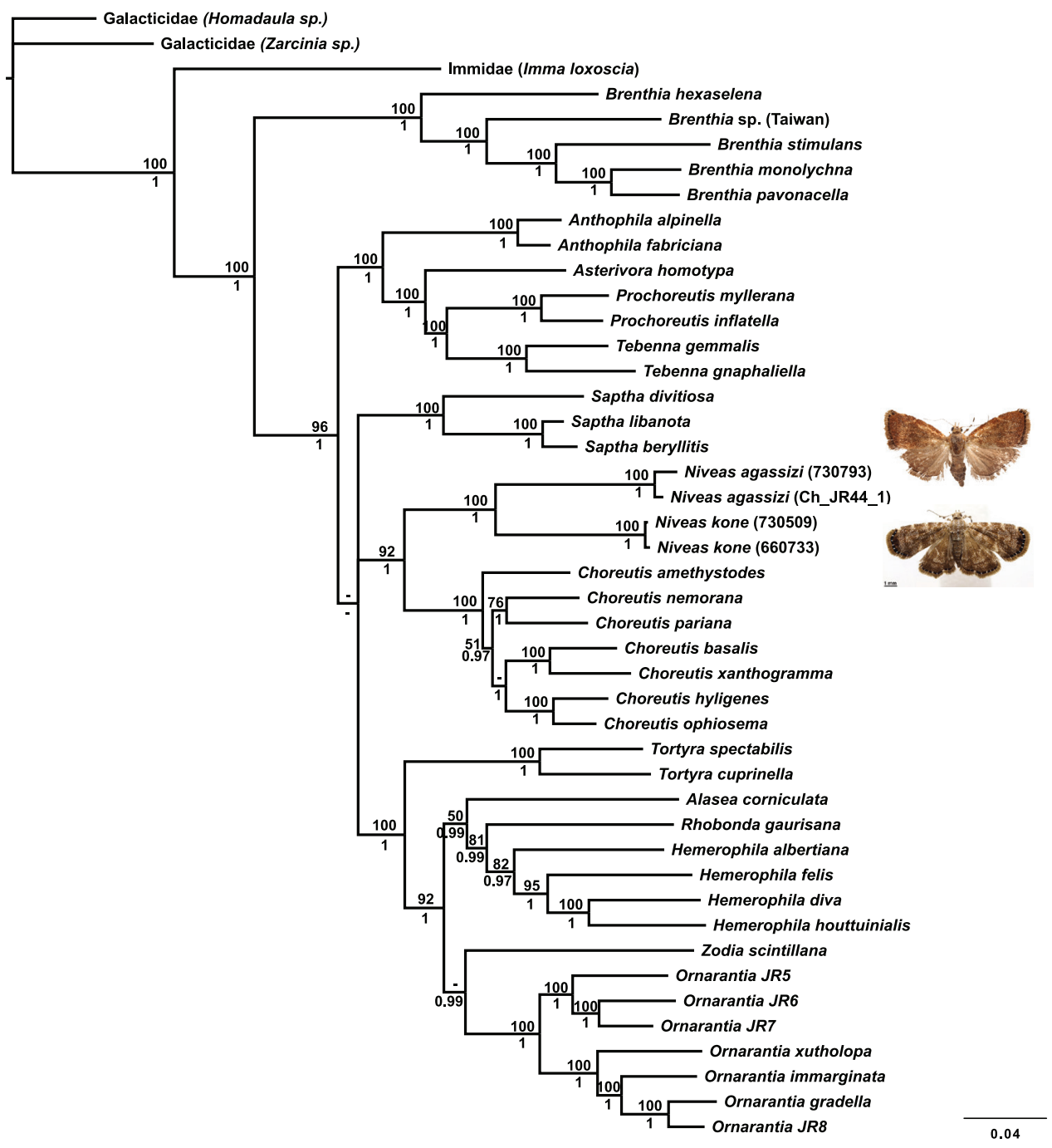

Figure I I. Phylogenetic tree from a Bayesian analysis showing the position of Niveas in relation to other choreutid genera. Maximum likelihood (ML) bootstraps are shown above branches, and Bayesian posterior probabilities (PP) are below branches; dashes represent $\mathrm{ML}$ bootstraps $<50$ and $\mathrm{PP}<0.95$.

larvae, which possess very long hairs (Rota 2008a). Project field notes indicate that the shelters are distinct from other local Choreutidae in having strong white webbing. BRC has encountered them most commonly in the lowland coastal areas around Madang (city), but also in the coastal mountains behind Madang (up to about $100 \mathrm{~m}$ elevation), and at Wanang in the Ramu River Basin $(115 \mathrm{~m})$. The species has been recorded in publications (e.g., supplement to Novotny et al. 2010) and online databases as TORT015, misidentified as Brenthia sp. Based on locality information provided by Taylor and Maffi (1978: 185, 212), the Solomon Islands specimens are from lowland and foothill localities near Honiara, Guadalcanal; they were collected in light traps. 


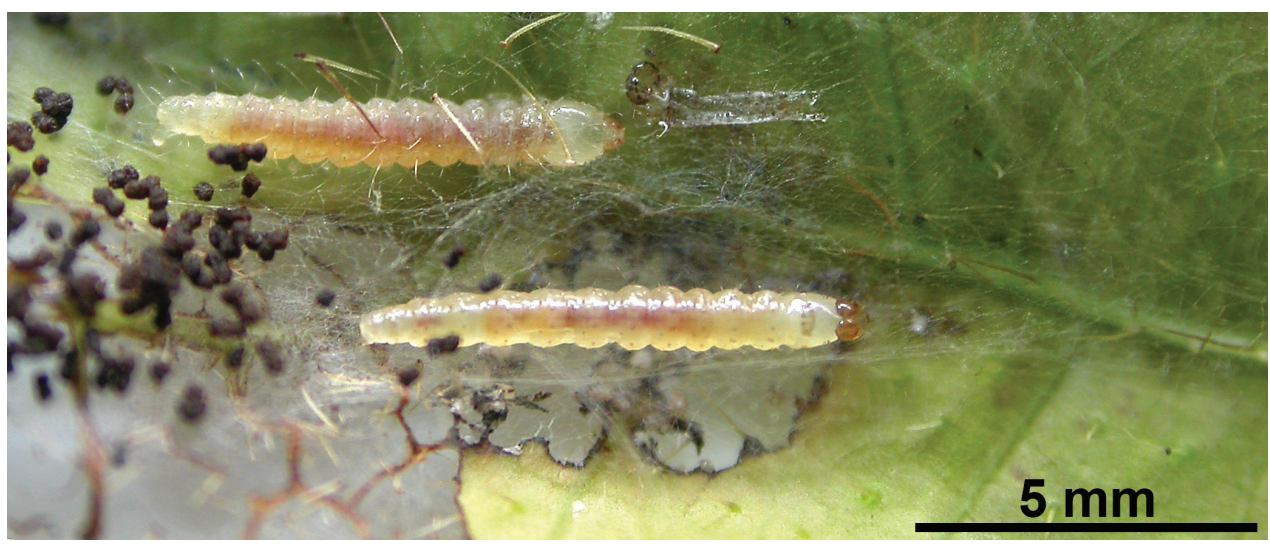

Figure I2. A photograph of the Niveas kone larvae made in the field.

Taxon descriptions are also organized in tabular format for ease of comparison (see Appendix).

\section{Discussion}

The two species of Niveas described herein are superficially quite different, but upon closer examination it becomes apparent that they share a number of morphological features. We consider the following as potential autapomorphies of Niveas: fusion or reduction in $\mathrm{R}$ veins in the forewing (Figs 5, 6); presence of round, concave sclerotizations on the A7 sternite in females (arrows in Figs 8, 9); strong lateral sclerotizations at the base of the ductus bursae (triangles in Figs 8, 9); and the presence of a terminal black band with white spots in the forewing (arrows in Figs 1, 3). In all other Choreutinae genera there are five fully-separated radial veins in the forewing; the A7 sternite in the female, as well as the base of the ductus bursae, are evenly sclerotized; and if present, a black terminal band in the forewing lacks white spots.

The split between $N$. kone and $N$. agassizi has presumably happened a long time ago based on the large COI divergence between them and the length of branches in the phylogenetic analysis including the nuclear genes. We considered assigning each species to its own monotypic genus because of their different external appearance, as well as some of the differences in venation and some aspects of female genitalia. It is unfortunate that $N$. agassizi is known from females only as perhaps the morphology of the male genitalia would help clarify the status of this species. However, we believe that $N$. kone and $N$. agassizi being each other's closest relatives among the currently known species of choreutids is best conveyed by assigning them to a single genus and therefore we opted for this more conservative approach. It is conceivable that other species of Niveas that might bridge this gap in both genetic and morphological variation will be discovered in the future. On the other hand, it is also possible that a new genus will need to be erected to accommodate $N$. agassizi and its currently unknown relatives. 


\section{Acknowledgements}

Papua New Guinea: This paper stems from a rearing campaign led by Vojtech Novotny, George Weiblen, Yves Basset, and Scott Miller, and supported by the US National Science Foundation (grants DEB-0211591, 0515678 and others), Czech Science Foundation grant 206/09/0115 and others, and Czech Ministry of Education \& European Union grant CZ.1.-07/2.3.00/20.0064. We thank the staff at the PNG Binatang Research Center for field assistance, PNG land owners for access to field sites and assistance, and PNG agencies for permits. DNA barcoding was provided by Paul Hebert through a grant from Genome Canada and the Ontario Genomics Institute in support of the iBOL project. Karolyn Darrow, Lauren Helgen and Margaret Rosati provided assistance at the Smithsonian. Kenya: We thank David Agassiz for sharing his material of African choreutids and the National Museums of Kenya for facilitating our collaboration. Phylogenetics: We thank Niklas Wahlberg for designing primers used in this project; and Carlos Peña and Eero Vesterinen for laboratory assistance. Phylogenetic analyses were conducted on the freely available Bioportal cluster (http://www. bioportal.uio.no) and RAxML blackbox (http://phylobench.vital-it.ch/raxml-bb/). We thank John Brown and an anonymous reviewer for helpful comments on an earlier version of the manuscript. SEM also thanks the Natural History Museum, London, Bishop Museum, Honolulu, and International Center for Insect Physiology and Ecology, Nairobi, for their continued support of this research program. JR was funded by the Finnish Kone Foundation experienced researcher grant during this project.

\section{References}

Craft KJ, Pauls SU, Darrow K, Miller SE, Hebert PDN, Helgen LE, Novotny V, Weiblen GD (2010) Population genetics of ecological communities with DNA barcodes: An example from New Guinea Lepidoptera. Proceedings of the National Academy of Sciences of the United States of America 107: 5041-5046. doi: 10.1073/pnas.0913084107

Cummins CA, McInerney JO (2011) A Method for Inferring the Rate of Evolution of Homologous Characters that Can Potentially Improve Phylogenetic Inference, Resolve Deep Divergence and Correct Systematic Biases. Systematic Biology 60: 833-844. doi: 10.1093/ sysbio/syr064

Diakonoff A (1986) Glyphipterygidae auctorum sensu lato: (Glyphiterygidae sensu Meyrick, 1913); Tortricidae: Hilarographini, Choreutidae, Brachodidae (partim), Immidae and Glyphipterygidae. G. Braun, Druckerei und Verlage, Karlsruhe, Plates Volume (175 pls.) +436 pp.

Hrcek J, Miller SE, Quicke DLJ, Smith MA (2011) Molecular detection of trophic links in a complex insect host-parasitoid food web. Molecular Ecology Resources 11: 786-794. doi: 10.1111/j.1755-0998.2011.03016.x 
Hrcek J, Miller SE, Whitfield JB, Shima H, Novotny V (2013) Parasitism rate, parasitoid community composition and host specificity on exposed and semi-concealed caterpillars from a tropical rainforest. Oecologia 173: 521-532. doi: 10.1007/s00442-013-2619-6

The Plant List (2010) Version 1. http://www.theplantlist.org/ [accessed August 5.2013]

Miller SE, Hrcek J, Novotny V, Weiblen GD, Hebert PDN (2013) DNA barcodes of caterpillars (Lepidoptera) from Papua New Guinea. Proceedings of the Entomological Society of Washington 115: 107-109. doi: 10.4289/0013-8797.115.1.107

Miller SE, Novotny V, Basset Y (2003) Studies on New Guinea moths. 1. Introduction (Lepidoptera). Proceedings of the Entomological Society of Washington 105: 1034-1042.

Nieukerken EJ van, Kaila L, Kitching IJ, Kristensen NP, Lees DC, Minet J, Mitter C, Mutanen M, Regier JC, Simonsen TJ, Wahlberg N, Yen S-H, Zahiri R, Adamski D, Baixeras J, Bartsch D, Bengtsson BÅ, Brown JW, Bucheli SR, Davis DR, De Prins J, De Prins W, Epstein ME, Gentili-Poole P, Gielis C, Hättenschwiler P, Hausmann A, Holloway JD, Kallies A, Karsholt O, Kawahara A, Koster JC, Kozlov M, Lafontaine JD, Lamas G, Landry J-F, Lee S, Nuss M, Park K-T, Penz C, Rota J, Schmidt BC, Schintlmeister A, Sohn JC, Solis MA, Tarmann GM, Warren AD, Weller S, Yakovlev RV, Zolotuhin VV, Zwick A (2011) Order Lepidoptera. In: Zhang Z-Q (Ed) Animal biodiversity: An outline of higher-level classification and survey of taxonomic richness. Zootaxa 3148: 212-221.

Novotny V, Miller SE, Baje L, Balagawi S, Basset Y, Cizek L, Craft KJ, Dem F, Drew RAI, Hulcr J, Leps J, Lewis OT, Pokon R, Stewart AJA, Samuelson GA, Weiblen GD (2010) Guildspecific patterns of species richness and host specialization in plant-herbivore food webs from a tropical forest. Journal of Animal Ecology 79: 1193-1203. doi: 10.1111/j.13652656.2010.01728.x

Peña C, Malm T (2012) VoSeq: A Voucher and DNA Sequence Web Application. PLoS ONE 7(6): e39071. doi: 10.1371/journal.pone.0039071

Rambaut A, Drummond AJ (2007) Tracer v1.4. http://beast.bio.ed.ac.uk/Tracer

Ratnasingham S, Hebert PDN (2007) BOLD: The Barcode of Life Data System (http:// www.barcodinglife.org). Molecular Ecology Notes 7: 355-364. doi: 10.1111/j.14718286.2007.01678.x

Ratnasingham S, Hebert PDN (2013) A DNA-Based Registry for All Animal Species: The Barcode Index Number (BIN) System. PLoS ONE 8(7): e66213. doi: 10.1371/journal. pone. 0066213

Ronquist F, Teslenko M, van der Mark P, Ayres DL, Darling A, Hohna S, Larget B, Liu L, Suchard MA, Huelsenbeck JP (2012) MrBayes 3.2: Efficient Bayesian Phylogenetic Inference and Model Choice Across a Large Model Space. Systematic Biology 61: 539-542. doi: $10.1093 /$ sysbio/sys029

Rota J (2005) Larval and pupal descriptions of the neotropical choreutid genera Rhobonda Walker and Zodia Heppner (Lepidoptera : Choreutidae). Annals of the Entomological Society of America 98: 37-47. doi: 10.1603/0013-8746(2005)098[0037:lapdot]2.0.co;2

Rota J (2008a) Immature stages of metalmark moths from the genus Brenthia Clemens (Lepidoptera: Choreutidae): morphology and life history notes. Journal of the Lepidopterists Society 62: 121-129. 
Rota J (2008b) A new genus and new species of metalmark moths (Lepidoptera: Choreutidae) from Costa Rica. Zootaxa 1933: 12-18.

Rota J (2011) Data partitioning in Bayesian analysis: molecular phylogenetics of metalmark moths (Lepidoptera: Choreutidae). Systematic Entomology 36: 317-329. doi: 10.1111/j.1365-3113.2010.00563.x

Rota J, Wahlberg N (2012) Exploration of data partitioning in an eight-gene data set: phylogeny of metalmark moths (Lepidoptera, Choreutidae). Zoologica Scripta 41: 536-546. doi: 10.1111/j.1463-6409.2012.00551.x

Stamatakis A, Hoover P, Rougemont J (2008) A rapid bootstrap algorithm for the RAxML web-servers. Systematic Biology 75: 758-771. doi: 10.1080/10635150802429642

Taylor B, Maffi M (1978) A review of the mosquito fauna of the Solomon Islands (Diptera: Culicidae). Pacific Insects 19: 165-248.

Wahlberg N, Wheat CW (2008) Genomic outposts serve the phylogenomic pioneers: designing novel nuclear markers for genomic DNA extractions of Lepidoptera. Systematic Biology 57: 231-242. doi: 10.1080/10635150802033006

Wilson JJ (2012) DNA barcodes for insects. In: Kress WJ, Erickson DL (Eds) DNA Barcodes: Methods and Protocols. Springer, New York, 17-46. doi: 10.1007/978-1-61779-591-6_3 


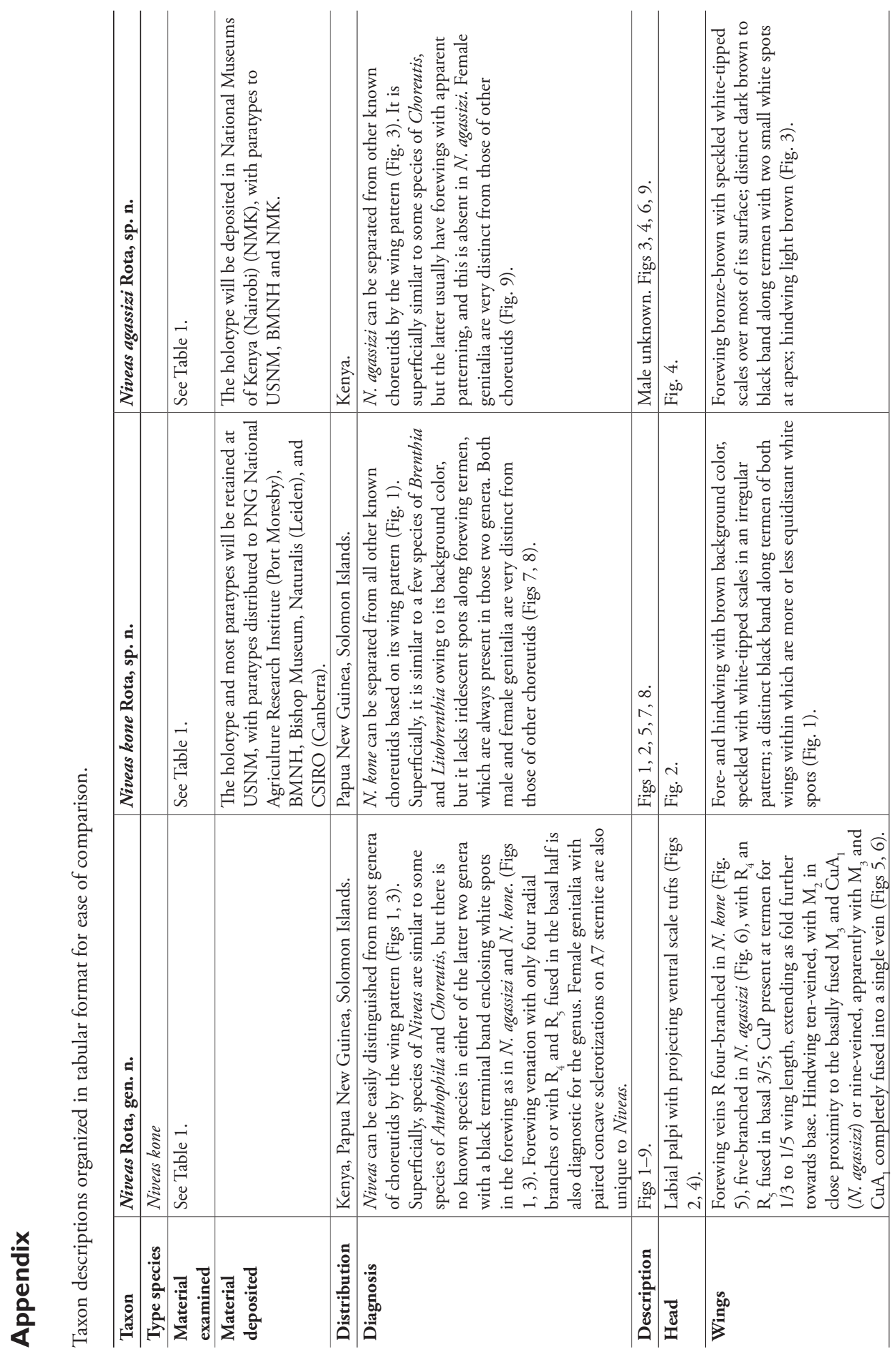




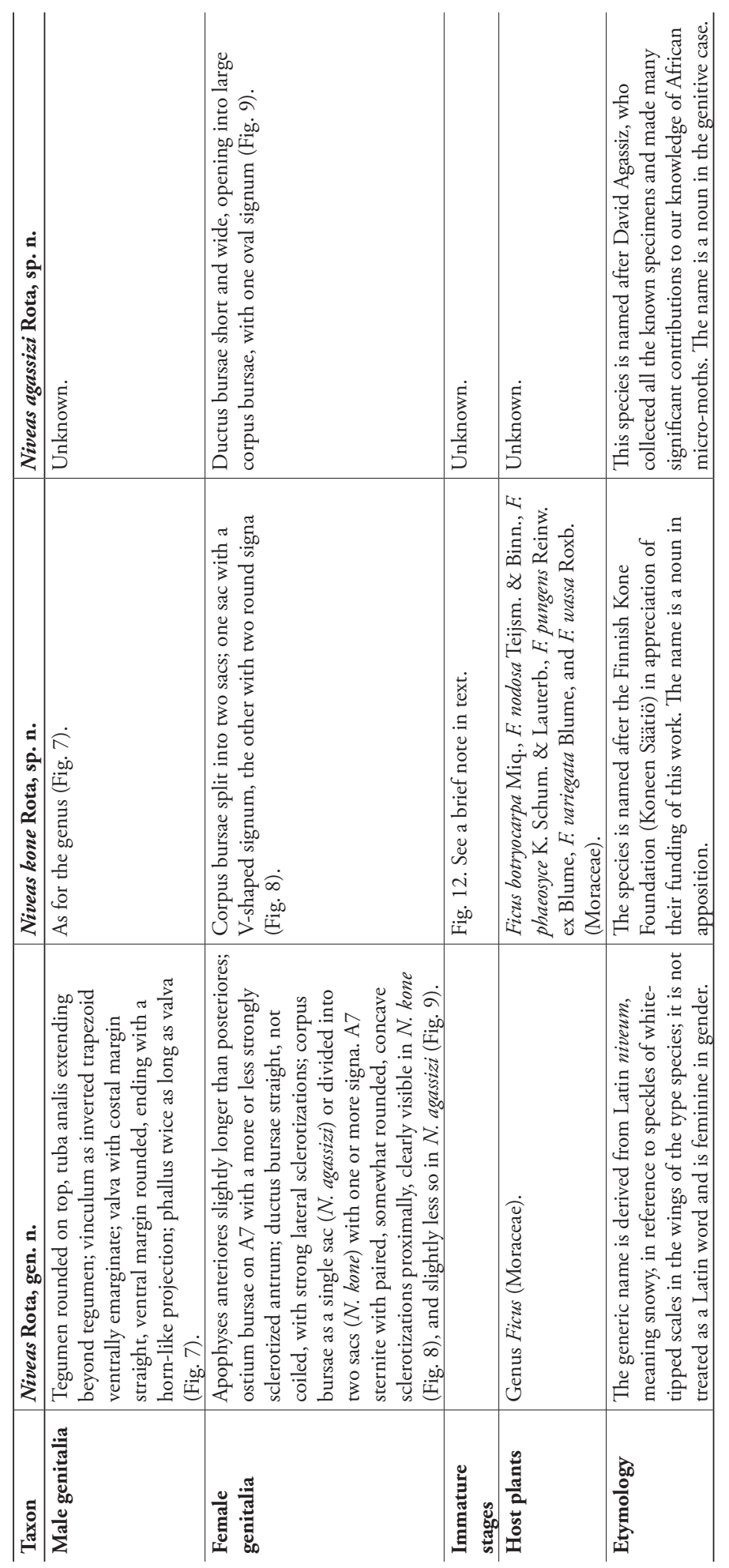

